\title{
Area di Accoglimento e Degenza Breve (ADB): parte integrante di un reparto di Medicina Interna organizzato per intensità di cure
}

\author{
Area of admission and short-term care: an integral part of the internal \\ medicine ward organized to intensive care
}

\section{Francesco Serafini *, Luca Bonanni, Andrea Zancanaro, Giuseppina Fricano, Marco Giannoccaro, Elisa Zulian, Michele Dalla Vestra, Paola Atanasio, Pierantonio Conton, Carlo De Riva, Giuseppe Fantin, Fabio Presotto}

\author{
UO Medicina Interna, Ospedale dell'Angelo, Mestre, Azienda ULSS 12 Veneziana
}

Ricevuto il 28 settembre 2011; accettato il 16 marzo 2012

disponibile online il 22 aprile 2012

\section{KEYWORDS \\ Medical admission; \\ Triage; \\ Short-term care; \\ Bedside echography.}

\begin{abstract}
Summary
Introduction: The analysis of the production related to health care activities in the Medicine ward of dell'Angelo Hospital (Mestre-Venezia, Italy) in the year 2010 reveals that the short-term hospitalization, less than 3 days, corresponds to $18 \%$ of all inpatient admissions.

Objectives: The short-term hospitalization approach need to be "governed", both in terms of effectiveness and efficiency. In our department we have identified an area called "Admission and Short Stay Area", where the discharge follows a comprehensive diagnostic and therapeutic pathway. Accordingly, we plan to extend the number of beds dedicated to the short stay in hospital, in order to decrease the average duration of stay avoiding the risk of increasing the readmissions, to share new pathways between health care workers.

Materials and methods: Within the department we have identified 8 beds for patients admitted from the emergency room. In the same beds triage is performed. In these beds the maximum duration of stay is 3 days. To achieve this goal we used clinical pathways shared between health workers, and bedside diagnostic procedures such as ultrasounds.

Results: In 2011 than to year 2010 there was an increased rate of short-term hospitalization (22-18\%). Despite that the mean hospital stay was unchanged (10.3-10.6 days). The number of readmission within 90 days was also unchanged when we considered the most common diseases. More than $90 \%$ of health care workers followed the pre-established clinical care pathways.
\end{abstract}

\footnotetext{
* Corrispondenza: UO di Medicina Interna, Ospedale dell'Angelo, via Paccagnella 11/a - 30174 Venezia.

E-mail: serafini.france@gmail.com (F. Serafini).
} 
Conclusions: The presence of a small number of beds within the medicine ward was dedicated to perform triage that allows to identify clinical care the needs of the patient. Among these some can be resolved in 3 days, rewarding patients and saving human and financial resources. To achieve this goal it is necessary that health care providers share clinical pathways, and that the bedside ultrasound is accessible.

(c) 2012 Elsevier Srl. All rights reserved.

\section{Introduzione}

L'analisi della produzione sanitaria riferita all'attività dell'Unità Operativa di Medicina Interna dell'Ospedale dell'Angelo (Mestre-Venezia) per l'anno 2010 rivela che la degenza di breve durata, inferiore a 3 giorni, corrisponde al 18\% di tutti i ricoveri ordinari. Risultati analoghi, anche se meno consistenti, si osservano nei reparti di Geriatria e di Nefrologia. Questo tipo di ricovero si confonde con l'Osservazione Breve Intensiva (OBI) del Pronto Soccorso. Tuttavia, la degenza breve si discosta dall'OBI sia per il tempo di ricovero (72 ore) sia per la gravità della casistica; in particolare, il malato presenta instabilità medio-bassa, richiede un monitoraggio iniziale e l'esecuzione di esami o procedure in tempi molto stretti.

Il ricovero breve, per essere tale, deve allora rispondere a criteri di efficienza e di efficacia: l'efficienza per escludere quella casistica che potrebbe essere ricondotta all'OBI (24 ore) e per includere degenze considerate più lunghe; l'efficacia affinché la dimissione consegua a un percorso diagnostico e terapeutico esauriente scongiurando un rientro a breve distanza di tempo. Quest'ultimo obiettivo è raggiungibile utilizzando percorsi clinico-assistenziali semplici, ripetibili e condivisi tra tutti gli operatori sanitari. La diagnostica "leggera", come l'ecografia al letto del malato ("bedside echography”), è sicuramente lo strumento più semplice e completo.

Nell'Unità Operativa di Medicina Interna dell'Ospedale dell'Angelo esiste da tempo un'organizzazione per intensità di cure che prevede, dopo un primo triage medico-infermieristico, l'allocazione del paziente nei letti di Medicina subintensiva (6), nei letti per post-acuti (15), nei letti di degenza ordinaria (55). Nel 2011 abbiamo individuato 8 posti letto da destinare sia all'Accoglimento per il Pronto Soccorso sia alla Degenza Breve (ADB). In questa maniera ci siamo proposti di aumentare, dopo adeguata selezione, la percentuale di dimissioni brevi (entro 72 ore dall'ingresso in reparto), di mantenere la degenza media rispetto agli standard dell'anno precedente, di implementare e condividere i percorsi clinicoassistenziali. Abbiamo misurato la qualità del lavoro in Degenza Breve monitorando i rientri per le malattie più comuni e confrontandoli con quelli del 2010. Tutto il lavoro è stato ideato e realizzato con isorisorse.

\section{Materiali e metodi}

\section{Pazienti}

Tutti i pazienti provenienti dal Pronto Soccorso (99\% dei ricoveri) passavano per l'area $A D B$. Qui medico e infermiere effettuavano un primo triage, clinico-assistenziale, utilizzando scale già consolidate in letteratura: il Modified Early Warning Score (MEWS), l'indice di Barthel e l'Indice di Complessità Assistenziale (ICA) [1-3]. A seconda della gravità clinica e della complessità gestionale il paziente veniva quindi allocato in una delle tre aree a diversa intensità di cura: subintensiva, post-acuti, degenza ordinaria (tab. 1). I pazienti aventi caratteristiche congrue con la degenza breve rimanevano nell'area ADB fino alla dimissione (entro 72 ore). Per questo scopo i pazienti dovevano presentare una bassa criticità clinica e una delle diagnosi riportate in tabella 2 .

\section{Area di Accoglimento e Degenza Breve}

Si tratta di due stanze similari e adiacenti, ciascuna comprendente: quattro letti dotati di sistema erogante ossigenoterapia, una postazione di osservazione con computer collegato alla rete, un monitor per la misurazione continua di pressione arteriosa, frequenza cardiaca, saturazione di ossigeno. A disposizione dell'area $A D B$ vi sono inoltre un carrello per le urgenze, un sistema di ventilazione meccanica a pressione positiva delle vie aeree (CPAP), un defibrillatore semiautomatico, un ecografo portatile con sonde per cuore, vasi e addome.

Quivi erano sempre presenti un medico (dalle 13 alle 20 il medico di guardia) e due infermieri. I trasferimenti nelle altre aree del reparto erano condivisi con il personale che accoglieva il malato.

\section{Strumenti}

Medico e infermieri dell'area ADB si avvalevano fondamentalmente di due strumenti: i percorsi clinico-assistenziali e la bedside echography.

I percorsi erano stati messi a punto da tutti gli operatori sanitari nel corso del 2010-2011 attraverso la formazione continua sul campo e riassumevano le competenze dei sanitari (medico, infermiere, operatore sanitario) in presenza di quattro sintomatologie: dispnea, dolore toracico, dolore addominale, febbre. La "tabella delle competenze" era articolata in tre intervalli di tempo: l'accoglimento, le prime 12 ore, le successive 36 ore.

Il medico, inoltre, utilizzava un ecografo mobile dotato di tre sonde: lineare per il vascolare, settoriale per il cuore e i polmoni, convessa per l'addome. La maggior parte dei medici dell'Unità Operativa era stata formata mediante corsi di bedside echography.

\section{Indicatori}

Il primo obiettivo era quello di aumentare le dimissioni a 3 giorni. Considerando che le caratteristiche dei pazienti 
Tabella 1 Pazienti/condizioni cliniche per le aree subintensiva, post-acuti e di degenza ordinaria.

\begin{tabular}{|c|c|c|}
\hline Subintensiva & Post-acuti & Degenza ordinaria \\
\hline $\begin{array}{l}\text { Pazienti per i quali: } \\
\text { - la terapia parenterale sia effettuata } \\
\text { continuativamente } \\
\text { - la misurazione dei parametri vitali } \\
\text { avvenga più volte nella giornata o su } \\
\text { valutazione del sanitario } \\
\text { - le indagini diagnostiche (elettivamente } \\
\text { di laboratorio) siano ripetute } \\
\text { frequentemente nella giornata }\end{array}$ & $\begin{array}{l}\text { Pazienti per i quali vi sia: } \\
\text { - necessità di controllo dei parametri } \\
\text { vitali non più di } 1 \text { volta al giorno } \\
\text { - necessità di controlli di laboratorio } \\
\text { non più di } 2 \text { volte la settimana } \\
\text { - possibilità di terapia parenterale ma } \\
\text { senza necessità di monitoraggio } \\
\text { ravvicinato } \\
\text { - manovre diagnostiche o terapeutiche } \\
\text { invasive non più di } 1 \text { volta la } \\
\text { settimana }\end{array}$ & $\begin{array}{l}\text { Pazienti che richiedono: } \\
\text { - l'esame clinico almeno } 1 \text { volta al giorno } \\
\text { - la misurazione dei parametri vitali } \\
\text { almeno } 1 \text { volta al giorno } \\
\text { - gli accertamenti diagnostici almeno } \\
2 \text { volte la settimana } \\
\text { - la terapia parenterale senza } \\
\text { monitoraggio continuo bensì ravvicinato } \\
\text { - indagini e terapie invasive almeno } \\
2 \text { volte la settimana }\end{array}$ \\
\hline $\begin{array}{l}\text { Tra queste situazioni cliniche sono } \\
\text { compresi: } \\
\text { - emorragia gastrointestinale } \\
\text { (score di Rockall > 6) } \\
\text { - stato di coma, sopore, agitazione } \\
\text { psicomotoria, confusione mentale } \\
\text { - desaturazione (saturazione ossigeno in } \\
\text { aria ambiente }<90 \% \text { ) } \\
\text { - pallore e sudorazione } \\
\text { - dolore toracico in fase di accertamento } \\
\text { (complicato e/o con comorbilità) } \\
\text { - emergenza ipertensiva } \\
\text { - IMA non Q non di competenza } \\
\text { cardiologica } \\
\text { - BPCO riacutizzata e insufficienza } \\
\text { respiratoria } \\
\text { - embolia polmonare con compromissione } \\
\text { emodinamica (BNP e troponina positivi) } \\
\text { - shock settico } \\
\text { - scompenso cardiaco acuto e cronico } \\
\text { (complicato e/o con comorbilità, Classe } \\
\text { di Killip > II) } \\
\text { - ictus cerebrale } \\
\text { - score MEWS } \geq 4\end{array}$ & $\begin{array}{l}\text { Il paziente che fin dall'ingresso o dopo } \\
\text { un periodo di cure nell'area } \\
\text { precedente può essere considerato } \\
\text { clinicamente stabile, ma non può } \\
\text { essere dimesso per problemi } \\
\text { socioassistenziali, deve essere } \\
\text { trasferito nell'area per post-acuti. } \\
\text { Tale paziente: } \\
\text { - necessita di essere inquadrato nel } \\
\text { percorso postdimissione } \\
\text { - ha un indice di Barthel compreso tra } \\
\text { 0 e } 30 \\
\text { - ha un'alta dipendenza assistenziale } \\
\text { - attende una sistemazione definitiva } \\
\text { in casa o struttura protetta }\end{array}$ & $\begin{array}{l}\text { Situazioni con livello di dipendenza basso- } \\
\text { intermedio (indice di Barthel > 30): } \\
\text { - stati infettivi senza segni di } \\
\text { compromissione emodinamica e } \\
\text { insufficienza multiorgano (endocardite, } \\
\text { pericardite, broncopolmonite, epatite, } \\
\text { colangite-colecistite, pancreatite, } \\
\text { pielonefrite, cistite, dermo- } \\
\text { epidermite...) } \\
\text { - malattia neoplastica in fase di diagnosi, } \\
\text { recidivante o resistente ma sempre } \\
\text { aggredibile con terapia convenzionale } \\
\text { (buon performance status) } \\
\text { - malattie reumatiche, autoimmuni, } \\
\text { endocrine in via di definizione } \\
\text { diagnostica } \\
\text { - anemie in via di definizione diagnostica } \\
\text { senza compromissione emodinamica }\end{array}$ \\
\hline
\end{tabular}

Legenda: IMA = infarto acuto del miocardio; BPCO = broncopneumopatia cronica ostruttiva; BNP = Brain Natriuretic Peptide; MEWS = Modified Early Warning Score.

ricoverati in Medicina Interna non rispecchiavano quelle della tabella delle competenze e che le diagnosi erano simili a quelle dell'OBI, la sfida principale consisteva nell'aumentare le dimissioni brevi di circa il 10\% rispetto al 2010.

Il secondo obiettivo era quello di mantenere una degenza media pari o inferiore a quella del 2010. L'incremento dei ricoveri atteso per il 2011, la chiusura della Lungodegenza e dell'Hospice rendevano improbabile il mantenimento degli standard precedenti (variazione attesa \pm 0-1 giorni).

Infine, il numero di rientri a distanza di 90 giorni dalla dimissione non doveva aumentare rispetto al 2010. In particolare, volevamo scongiurare un incremento percentuale delle riammissioni per le patologie prese in considerazione in area ADB: scompenso di cuore, bronchite cronica riacutizzata, fibrillazione atriale, embolia polmonare, diabete mellito. La variazione attesa era tra lo $0 \%$ e il $5 \%$.

Tutte le variabili qualitative, espresse in percentuali, venivano confrontate con il test del $\chi^{2}$ test o il test di Fisher.

\section{Risultati}

I dati sono estrapolati dall'analisi del periodo di tempo compreso tra gennaio e ottobre di entrambi gli anni 2010 e 2011.

Il numero di ricoveri ordinari di durata inferiore a 3 giorni è aumentato significativamente nel $2011\left(18,1 \% ; \chi^{2}=13,29\right.$; $\mathrm{p}<0,001$; IC $95 \% 20-24 \%$ vs $17-20 \%$ ) anche dopo aver escluso $i$ decessi, grazie alla creazione dell'area ADB.

L'utilizzo del triage e dei criteri di eleggibilità ha permesso di dimettere nei tempi prestabiliti la quasi totalità dei pazienti ricoverati in ADB. L'adesione da parte dei sanitari al percorso clinico-assistenziale, strumento di lavoro in $A D B$, è stata quasi totale, come dimostrato dal rapporto tra numero dei ricoveri e numero delle tabelle delle competenze compilate (tab. 3).

Rispetto al 2010, nel 2011 i ricoveri sono aumentati (+1,5\%; $p=$ non significativo; IC 95\% 2.076-3.232). Come negli anni precedenti, e nel rispetto degli obiettivi di budget del Dipartimento, i ricoveri ordinari sono stati quasi la totalità, 
Tabella 2 Valutazione dei pazienti per la degenza breve.

\section{Pazienti eleggibili}

Valutazione diagnostica e trattamento di un paziente con patologia acuta a bassa criticità clinica (score MEWS <4) ma con elevata complessità potenziale:

- attacco ischemico transitorio di lunga durata a rischio medio-basso

- trauma cranico commotivo (anche con piccola emorragia subaracnoidea)

- trauma costale anche fratturativo senza contusione polmonare

- sincope preceduta da prodromi

- colica addominale non chirurgica

- subocclusione intestinale

- fibrillazione atriale (sia per "rythm control” sia per "rate control")

- dolore toracico anteriore con score TIMI elevato

- polmonite di comunità di Classe III di Fine

- bronchite cronica con lieve riacutizzazione (saturazione ossigeno $>90 \%$ )

- alterazioni dell'equilibrio idroelettrolitico di lieve-media entità

- scompenso cardiaco non in prima diagnosi (Classe I-II di Killip alla dimissione dal Pronto Soccorso)

- sospetto di emorragia gastrointestinale con score di Rockall $<6$

- trombosi venosa profonda e tromboembolia polmonare minore (in compenso di circolo, troponina e BNP negativi)

- iperglicemia

Legenda: MEWS = Modified Early Warning Score; TIMI = Thrombolysis In Myocardial Infarction; BNP = Brain Natriuretic Peptide.

Tabella 3 Indicatori dell'area ADB (da gennaio a ottobre).

\begin{tabular}{lcccc}
\hline & $\begin{array}{c}\text { Numero di ricoveri } \\
\text { di durata }<3 \text { giorni } \\
\text { (esclusi i decessi) }\end{array}$ & $\begin{array}{c}\text { Ricoveri } \\
\text { totali \% (IC 95\%) }\end{array}$ & $\begin{array}{c}\text { Dimissioni/Ricoveri } \\
\text { in ADB } \\
\%\end{array}$ & $\begin{array}{c}\text { Ricoveri/Tabelle } \\
\text { di competenza in ADB } \\
\%\end{array}$ \\
\hline 2010 & 486 & $18(17-20 \%)$ & 90 & 90 \\
\hline Incremento (\%) & 574 & $22(20-24 \%)$ & & \\
& $\begin{array}{l}+18,1 \% \\
x^{2}=13,29 \\
\mathrm{p}<0,001\end{array}$ & & & \\
\hline
\end{tabular}

rimanendo i programmati ben sotto la soglia del 5\% (tab. 4). Il tasso di occupazione nel 2011 è rimasto invariato rispetto all'anno precedente $(102,89$ vs 101,99$)$. Invariate sono state anche le diagnosi più frequenti di ricovero, rimanendo lo scompenso cardiaco al primo posto seguito dalla broncopneumopatia cronica ostruttiva. Le neoplasie hanno uguagliato al terzo posto le malattie cerebrovascolari a causa della chiusura della Lungodegenza e dell'Hospice.

La degenza media è rimasta costante rispetto al 2010 (IC $95 \% 10,2-10,4 \%$ vs $10,5-10,7 \%$ ), mentre i decessi sono apparsi lievemente ridotti ( $\mathrm{p}=$ non significativo; IC 95\% 9,8$12 \%$ vs $11-13 \%)$. Se $i$ valori verranno confermati fino alla fine del 2011, la degenza media in Medicina Interna registrerà una sensibile riduzione $(10,3$ giorni vs 11,3$)$. La necessità di completare l'iter diagnostico in breve tempo, come richiesto per le patologie prese in considerazione, ha comportato un
In ragione della prospettiva di dimettere il paziente, se le condizioni cliniche lo consentono, entro 72 ore sono esclusi dal ricovero in area di Accoglimento e Degenza Breve:

- pazienti ad alta criticità clinica (score MEWS $\geq 4$ )

- pazienti ad alta dipendenza assistenziale (indice di Barthel $\leq 30$ )

- pazienti con malattia infettiva trasmissibile e/o che necessitino di isolamento per immunodeficienza

- pazienti terminali sensibile aumento degli esami ecografici al letto del malato $(+39 \%)$.

Il monitoraggio dei rientri a 90 giorni e oltre per le diagnosi prese in considerazione nell'area ADB non ha mostrato variazioni significative rispetto al 2010. Le riammissioni per scompenso cardiaco, bronchite cronica riacutizzata, fibrillazione atriale, diabete mellito, embolia polmonare mostrano addirittura un trend favorevole, sebbene non statisticamente significativo. I pazienti dimessi con diagnosi di occlusione intestinale sono rientrati più frequentemente.

\section{Discussione}

L'osservazione breve, nota anche come $\mathrm{OBI}$, è parte integrante del Pronto Soccorso e ha caratteristiche e procedure 
Tabella 4 Struttura dell'Ospedale dell'Angelo nel 2010.

\begin{tabular}{lllllll}
\hline & Posti letto (totali 523) & & Ricoveri (totali 19.000) & $\begin{array}{c}\text { Degenza media } \\
\text { nel 2010 (giorni) }\end{array}$ \\
\hline Area medica & 274 & Dipartimento di Medicina & & Dipartimento di Medicina & 4.000 & \\
\hline & & $\bullet$ Medicina Interna & 84 & $\bullet$ Medicina Interna & 2.845 & 11,3 \\
\hline & $\bullet$ Geriatria & 39 & $\bullet$ Geriatria & 730 & 12,5 \\
\hline Area chirurgica & 204 & Cardiologia & 16 & $\bullet$ Nefrologia & 425 & 1.600 \\
\hline Area materno infantile & 45 & & 29 & Cardiologia & 6,8 \\
\hline
\end{tabular}

ben standardizzate. Negli ultimi anni si è affermata l'esigenza di istituire un'area con simili funzioni anche nell'ambito dei reparti di Medicina Interna. In alcune Unità Operative è ormai consolidato il modello per intensità di cure, in grado di individuare e di offrire ai pazienti la necessaria terapia-assistenza, influendo così anche sulla durata della degenza [4].

La prima nozione di area di degenza breve proviene dall'esperienza americana degli "hospitalists", i medici ospedalieri. La creazione di una "hospital run medical short-stay unit" permetteva di selezionare i malati provenienti dal Pronto Soccorso e di trattarli durante una degenza media di 3 giorni [5].

L'area di degenza breve è accomunata con l'osservazione breve del Pronto Soccorso per un intervento tempo-correlato in presunzione della dimissione, ma ha maggiori analogie con la degenza ordinaria in quanto il paziente ha una pluripatologia, è spesso in una condizione di instabilità clinica ed è bisognoso di monitoraggio iniziale e di frequenti procedure o esami (intervento gravità-correlato). In quest'ottica risulta importante individuare sia i pazienti sia un'area dedicata alla loro cura.

Per quanto riguarda i pazienti abbiamo identificato, con l'aiuto della letteratura, alcune patologie a bassa criticità clinica che potevano avvalersi di una diagnostica rapida e "leggera" (tab. 2) [5,6]. La dimissione breve è stata infatti descritta per lo scompenso cardiaco non complicato (degenza media di 3,9 giorni) [7], per la bronchite cronica riacutizzata con adeguata saturazione (degenza media di 3,2 giorni) [8], per l'embolia polmonare in compenso emodinamico (degenza da 1 a 4 giorni) [9].

All'interno del nostro reparto abbiamo poi individuato un'area che fungesse sia da accoglimento per il Pronto Soccorso sia da degenza breve (ADB). Il personale sanitario, dedicato al lavoro nell'area, ha quindi messo a punto alcuni percorsi clinico-assistenziali ed è stato formato per eseguire l'ecografia al letto del malato. All'inizio del 2011 l'area era funzionante.

L'aumento percentuale delle dimissioni a 3 giorni $(+18 \%)$ ha permesso di fronteggiare l'aumento del numero dei ricoveri $(+1,5 \%)$ nel corso del 2011 . Le diagnosi di dimissione interessavano tutte le patologie individuate per l'ADB: in particolare $\mathrm{i}$ casi di diabete mellito $(+15 \%)$, disidratazione $(+14 \%)$, fibrillazione atriale $(+4 \%)$, scompenso cardiaco $(+3 \%)$ e bronchite cronica riacutizzata $(+6 \%)$.

La degenza media, nel periodo esaminato, non cambiava rispetto all'anno precedente $(10,3$ giorni verso 10,6$)$, anche se il trend sembra in miglioramento se rapportato a fine
2010 (11,3). Tra le cause vi era sicuramente l'aumento dei ricoveri per pazienti neoplastici terminali; infatti nel 2011 chiudevano 25 posti letto di Hospice e il numero dei pazienti oncologici afferenti alla nostra ASL raggiungeva la media di 600.

Per testare la qualità del lavoro abbiamo monitorato il numero di esami ecografici e i rientri a 90 giorni. Tenuto conto che per molte delle condizioni cliniche prescelte la diagnostica richiedeva una semplice ecografia, il numero degli esami era aumentato sia complessivamente sia per singolo distretto (cardiaco in primis, addominale e vascolare). Il risultato era ascrivibile al fatto che l'ecografia veniva praticata da più operatori e sempre al letto del malato.

Abbiamo monitorato i rientri a 90 giorni per le patologie scelte nell'area di degenza breve. Rispetto al 2010 i reingressi per tutte le diagnosi selezionate presentavano un trend favorevole senza raggiungere una significatività statistica. Il successo era da attribuirsi al lavoro multidisciplinare. I pazienti con scompenso cardiaco ricevevano opuscoli informativi, diete ad hoc e quando necessario venivano ricontattati telefonicamente $[10,11]$. Il paziente con bronchite cronica ostruttiva veniva affidato all'ambulatorio specialistico [12]. I pazienti diabetici e con fibrillazione atriale erano rivisti, se necessario, nell'ambulatorio del post-ricovero.

I risultati dello studio vanno interpretati con molta cautela. In particolare, i pazienti scelti sono quelli a bassa complessità e non corrispondono alla totalità dei ricoveri. Il rientro a 90 giorni è sicuramente un indicatore più debole rispetto alla mortalità. Alcune patologie hanno richiesto un eccesso di esami del sangue.

Attraverso un questionario di "gradimento" distribuito a tutti i sanitari sono emerse poi alcune criticità. In particolare il rapido turnover dei letti ha richiesto un notevole lavoro fisico. Durante i giorni festivi e nelle "guardie" coperte dai medici di altri reparti i ricoveri non seguivano il percorso prestabilito. Infine, ma più sentita, era la necessità di potenziare l'organico del comparto. L'infermiere dedicato all'ADB doveva infatti accogliere tutti i ricoveri dal Pronto Soccorso, impostare il percorso clinico, trasferire i pazienti nelle aree di destinazione, monitorare quelli dimissibili a 3 giorni.

\section{Conclusioni}

In un reparto medico che opera secondo un modello per “intensità di cure", la presenza di un'area che funge da triage ma anche da degenza breve permette un migliore utilizzo delle risorse umane e tecnologiche, una riduzione 
della degenza media per alcune patologie ben identificate, un'adeguata terapia senza peggiorare la prognosi a breve termine.

Per ottenere questi risultati appare necessario condividere i percorsi clinici tra i sanitari, interagire con il Pronto Soccorso, avere a disposizione e saper utilizzare l'ecografia "bedside".

In un prossimo futuro è ipotizzabile che l'area ADB sia condivisa tra Unità Operative dello stesso Dipartimento, sia funzionante anche nei giorni festivi e sia dotata di personale sanitario adeguato per numero e per formazione.

\section{Conflitto di interessi}

Gli autori dichiarano di non aver nessun conflitto di interessi.

\section{Bibliografia}

1. Subbe $C P$, Kruger $M$, Rutherford $P$, Gemmel L. Validation of a modified Early Warning Score in medical admissions. QJM 2001;94(10):521-6.

2. Bartolomei C, Cei M. L'allocazione dei pazienti in un reparto di Medicina Interna organizzato per intensità di cure: lo studio ADOIT Tri-Co (Triage di Corridoio). It J Med 2007;1(2):31-9.

3. Volpe C, Bassan F, Bordin P, Castellano D, Treppo G, Moretti V. Il paziente postacuto: significato di una definizione. It J Med 2007;1(4):50-4.
4. Chesi G, Boni F. Ospedali e modelli organizzativi per intensità di cure: il punto di vista dell'internista. It J Med 2012;6(1): 63-71.

5. Abenhaim HA, Kahn SR, Raffoul J, Becker MR. Program description: a hospitalist-run, medical short-stay unit in a teaching hospital. CMAJ 2000;163(11):1477-80.

6. Lucas BP, Kumapley R, Mba B, Nisar I, Lee K, Ofori-Ntow S, et al. A hospitalist-run short-stay unit: features that predict length-ofstay and eventual admission to traditional inpatient services. J Hosp Med 2009;4(5):276-84.

7. Valle R, Canali C, Giovinazzo P, D’Atri M, Milani L. Is early discharge possible in patients with uncomplicated heart failure? Cost-efficacy analysis. Ital Heart J Suppl 2003;4(12): 965-72.

8. Cotton MM, Bucknall CE, Dagg KD, Johnson MK, MacGregor G, Stewart C, et al. Early discharge for patients with exacerbations of chronic obstructive pulmonary disease: a randomized controlled trial. Thorax 2000;55(11):902-6.

9. Davies CW, Wimperis J, Green ES, Pendry K, Killen J, Mehdi I, et al. Early discharge of patients with pulmonary embolism: a two-phase observational study. Eur Respir J 2007;30(4): 708-14.

10. McAlister FA, Stewart S, Ferrua S, McMurray JJ. Multidisciplinary strategies for the management of heart failure patients at high risk for admission: a systematic review of randomized trials. J Am Coll Cardiol 2004;44(4):810-9.

11. Joynt KE, Jha AK. Who has higher readmission rates for heart failure, and why? Implications for efforts to improve care using financial incentives. Circ Cardiovasc Qual Outcomes 2011;4(1): 53-9.

12. Cooper S. Early supported discharge at exacerbation of COPD: safe and cost effective for some. Thorax 2004;59(10):869. 